\title{
Pediatria do Neurodesenvolvimento em Portugal: \\ Movimento Hospitalar Assistencial, Recursos e Necessidades - Evolução em Dez Anos
}

\section{Neurodevelopmental Pediatrics in Portugal: Hospital Patient Volume, Resources and Needs - Changes After Ten Years}

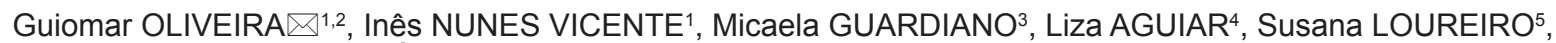
Rosa GOUVEIA ${ }^{6}$, Filipe GLÓRIA SILVA ${ }^{7}$

Acta Med Port 2021 Mar;34(3):185-193 - https://doi.org/10.20344/amp.13316

\section{RESUMO}

Introdução: As perturbações do neurodesenvolvimento são, nas sociedades modernas, as patologias crónicas mais frequentes da idade pediátrica. Muitas permanecem na vida adulta. A organização da rede de cuidados de saúde nacional carece de conhecimento fundamentado das necessidades assistenciais para lhes responder de um modo eficaz, eficiente e efetivo. Com o objetivo de conhecer a realidade assistencial hospitalar atual da Pediatria do Neurodesenvolvimento, a Sociedade de Pediatria do Neurodesenvolvimento da Sociedade Portuguesa de Pediatria procedeu a um levantamento nacional em 2007, repetindo-o dez anos depois.

Material e Métodos: No biénio 2016-2017 procedeu-se a um inquérito dirigido ao universo de 45 unidades hospitalares abrangendo o movimento assistencial das consultas de Pediatria do Neurodesenvolvimento, a alocação de recursos humanos, e as necessidades de reforço de profissionais.

Resultados: Obteve-se $100 \%$ de respostas. O número total de consultas de Pediatria do Neurodesenvolvimento subiu de 38238 (2007) para 99815 (2017). O número de profissionais também aumentou: os pediatras passaram de 82 a 156 . Uma mediana de 101 crianças aguardavam primeira consulta, contra 185 em 2007.

Discussão: Numa década, o movimento assistencial quase triplicou. O reforço de profissionais, apesar de positivo, não teve o mesmo incremento; ainda assim, o número de crianças em lista de espera para primeira consulta reduziu-se quase para metade, o que reflete o comprometimento dos profissionais.

Conclusão: É de salientar a melhoria global da resposta nacional na área da Pediatria do Neurodesenvolvimento. Contudo, o reforço dos recursos humanos numa perspectiva pluridisciplinar não deve ser negligenciado, tendo em vista a melhoria contínua da prestação de cuidados numa área de grande cronicidade e complexidade.

Palavras-chave: Hospitais; Pediatria; Perturbações do Neurodesenvolvimento; Portugal; Recursos em Saúde

\section{ABSTRACT}

Introduction: Neurodevelopmental disorders are, in modern societies, the most common chronic pediatric conditions. Many remain in adulthood. Organizing the national health care network to respond efficiently and effectively requires grounded knowledge of care needs. The Neurodevelopmental Pediatrics Society of the Portuguese Society of Pediatrics in order to know the current hospital care reality of Neurodevelopmental Pediatrics, carried out a national survey in 2007, repeating it ten years later.

Material e Methods: In the 2016-2017 biennium, a survey of 45 hospital units was conducted on the patient volume of Neurodevelopmental clinics, the allocation of human resources, and the needs for professional reinforcement.

Results: We obtained a $100 \%$ response rate. The total number of Neurodevelopmental Pediatrics consultations rose from 38238 (2007) to 99815 (2017). The number of professionals has also increased: pediatricians increased from 82 to 156 . A median of 101 children were awaiting first consultation, compared with 185 in 2007.

Discussion: In a decade, the patient volume almost tripled. The reinforcement of professionals, even though it was beneficial, did not increase accordingly; even so, the number of children on the waiting list for their first appointment has almost halved, reflecting the commitment of professionals.

Conclusion: It is noteworthy that the overall improvement of the national response in the area of Neurodevelopmental Pediatrics is remarkable. However, the requested reinforcement of human resources from a multidisciplinary perspective should not be neglected in view of the continuous improvement in care delivery in an area of great chronicity and complexity.

Keywords: Health Resources; Hospitals; Neurodevelopmental Disorders; Pediatrics; Portugal

\footnotetext{
1. Serviço do Centro de Desenvolvimento da Criança. Centro de Investigação e Formação Clínica. Hospital Pediátrico. Centro Hospitalar e Universitário de Coimbra. Coimbra. Portugal.

2. Clínica Universitária de Pediatria. Faculdade de Medicina. Universidade de Coimbra. Coimbra. Portugal.

3. Serviço de Pediatria. Unidade de Neurodesenvolvimento. Centro Universitário Hospitalar de São João. Porto. Portugal.

4. Serviço de Pediatria. Unidade de Neurodesenvolvimento. Hospital de Santarém. Santarém. Portugal.

5. Serviço de Pediatria. Unidade de Neurodesenvolvimento. Hospitalar Tondela-Viseu. Viseu. Portugal.

6. Serviço de Pediatria. Centro de Neurodesenvolvimento Infantil Logicamentes. Lisboa. Portugal.

7. Serviço de Pediatria. Consulta de Neurodesenvolvimento. Hospital CUF Sintra. Sintra. Portugal.

$\triangle$ Autor correspondente: Guiomar Oliveira. quiomar@chuc.min-saude.pt

Recebido: 20 de dezembro de 2019 - Aceite: 22 de maio de 2020 - First published: 26 de novembro de 2020 - Online issue published: 01 de março de 2021 Copyright $\odot$ Ordem dos Médicos 2021
} 


\section{INTRODUÇÃO}

As perturbações do neurodesenvolvimento, pela sua elevada e crescente frequência, cronicidade, complexidade e especificidade, carecem de uma organização da prestação de cuidados de forma estruturada e adaptada às necessidades atuais. ${ }^{1-3}$

Em Portugal, este percurso tem vindo a fazer-se paulatinamente. Há dez anos, sob a égide e coordenação da Sociedade de Pediatria do Neurodesenvolvimento da Sociedade Portuguesa de Pediatria (SPND-SPP), publicou-se o primeiro levantamento nacional sobre o movimento assistencial hospitalar, os recursos profissionais atribuídos a esta área, bem como os problemas existentes e as necessidades percecionadas relativamente ao ano de $2007 .{ }^{4}$

As conclusões então alcançadas, com a representação de $85,7 \%$ dos hospitais inquiridos, foram as seguintes: as consultas de desenvolvimento/neurodesenvolvimento representavam $11 \%$ da totalidade das consultas de Pediatria hospitalar; dedicavam-se a esta área diferenciada da Pediatria 82 pediatras (um terço a tempo completo); outros profissionais (psicólogos, terapeutas da fala, terapeutas ocupacionais, fisioterapeutas, técnicos de psicomotricidade, docentes e técnicos de serviço social) integravam estas equipas pluridisciplinares, mas em muito menor número, e só raramente o faziam a tempo completo. Estes recursos foram considerados insuficientes tendo em consideração a mediana do tempo de espera para consulta de seis meses, bem como o número elevado de crianças (mediana de 185 crianças) que se encontrava a aguardar a primeira consulta de pediatria do desenvolvimento/neurodesenvolvimento. ${ }^{4}$

Desde então, muitas alterações se têm constatado nesta área, sobretudo no que diz respeito à frequência e à concetualização diagnóstica e social das doenças pediátricas, bem como à elevada procura de cuidados de saúde hospitalares altamente diferenciados.

Até meados do século passado, as doenças agudas eram as dominantes e prevalecia o modelo biomédico. As doenças crónicas mais frequentes eram doenças 'físicas', como a asma e outras doenças respiratórias. Por motivos ainda não totalmente compreendidos, nas últimas décadas tem-se assistido a um aumento relativo das situações clínicas que se expressam como alterações do comportamento, das emoções, da aprendizagem e da capacidade de adaptação social. Desenvolveu-se então a perspetiva biopsicossocial da incapacidade ou disfunção adaptativa como expressão de menor saúde na criança, que motivou a Organização Mundial de Saúde (OMS) a criar a Classificação Internacional de Funcionalidade e Incapacidade em Saúde. ${ }^{5}$

Nos Estados Unidos da América (EUA), a prevalência de condições crónicas relacionadas com doença 'física' diminuiu $11,8 \%$ entre 2001 e 2011 , enquanto as associadas com problemas de neurodesenvolvimento e de saúde mental aumentaram 20,9\%. ${ }^{6}$ Há ainda evidência de que a limitação da capacidade adaptativa das crianças ao seu meio ambiente natural, por condições crónicas deste âmbito, quadruplicou de 1960 a 2009. ${ }^{5}$ Neste domínio, regista-se o aumento do diagnóstico dos problemas de linguagem e de aprendizagem, da perturbação do desenvolvimento intelectual, da perturbação do espetro do autismo, e do défice de atenção e hiperatividade, entre outros problemas de comportamento e emocionais. ${ }^{6}$

Este aumento não se explica somente pelo avanço nas tecnologias da saúde e pela melhoria da qualidade de vida e dos cuidados de saúde oferecidos às populações, que permitiram a sobrevivência de crianças com doenças anteriormente fatais. Outras variáveis estarão em causa para além destas. ${ }^{7}$

Os atuais modelos de saúde e sociais, orientados para uma monitorização apertada da normalidade e promotoras de rastreios precoces das doenças, podem eventualmente promover o diagnóstico de condições clínicas que no passado ficariam omissas (overutilization). ${ }^{8}$ Há autores que apelidam negativamente este período de 'too much medicine'. ${ }^{9}$

No contexto das patologias do neurodesenvolvimento, estas questões podem ser ainda mais relevantes, no sentido em que não existem biomarcadores que possam auxiliar na afirmação ou negação precisa do diagnóstico. Esta conclusão baseia-se essencialmente na observação clínica e na informação veiculada pelos pais e educadores. Acresce ainda que a variabilidade normal do comportamento e das etapas de aquisição do neurodesenvolvimento é muito ampla e nem sempre conhecida e valorizada pelos pais e profissionais, o que pode levantar preocupações por vezes infundadas. Por outro lado, o foco social atual numa aprendizagem padronizada imprime nas crianças com características cognitivas e comportamentais nos limites inferiores da normalidade um risco acrescido de insucesso escolar e, em consequência, a necessidade de cuidados de saúde no domínio do neurodesenvolvimento. Entre outros, estes fatores poderão contribuir para explicar a circunstância atual de se assistir a um aumento do diagnóstico das doenças do neurodesenvolvimento, emocionais e comportamentais, sobretudo em crianças provenientes de famílias em vantagem social, nas quais se tem verificado um incremento mais acentuado. ${ }^{6}$

Nesta realidade epidemiológica, e com o objetivo de uniformizar os critérios de diagnóstico, e de definir entidades clínicas específicas e discretas que com frequência coexistem, a Associação Americana de Psiquiatria, na sua quinta edição do Diagnostic and Statistical Manual of Mental Disorders (DSM), introduziu pela primeira vez o capítulo das perturbações do neurodesenvolvimento (neurodevelopmental disorders). ${ }^{10}$ Esta abordagem da disfunção das capacidades de aprendizagem e adaptativas na progressão do desenvolvimento psicomotor traz consigo o conceito de organicidade neurológica. Esta tem início precocemente e mantem-se por toda a vida, embora com espetro de manifestações clínicas muito diferente ao longo do tempo. ${ }^{2}$ Estas perturbações passaram assim a ser consideradas físicas ou orgânicas, tal como as demais que afetam outros órgãos que não o sistema nervoso. ${ }^{11}$ 
Reconhecendo a premência da investigação etiológica e da compreensão dos circuitos neuronais anómalos que Ihe estão subjacentes, o National Institute of Mental Health dos Estados Unidos da América lançou recentemente o Research Domain Criteria (RDoC). Este projeto de investigação preconiza uma estrutura de estudo científico que liga a vertente dimensional aos diferentes domínios da investigação biológica, por grupos semiológicos. Este modelo tem a vantagem de não tornar necessária a classificação em condições clínicas específicas, o que pode facilitar a análise e reduzir os enviesamentos científicos decorrentes de erros de diagnóstico em entidades clínicas discretas ou categóricas. ${ }^{12}$

Neste enquadramento de grande especificidade, os serviços de saúde, particularmente na especialidade de Pediatria, têm que se preparar para responder às necessidades das crianças, das famílias, das equipas de profissionais da educação, da saúde e dos investigadores em neurociência. Esta necessidade de análise, de preparação de profissionais e de definição do melhor modelo de resposta em saúde é discutida e abordada em todo o mundo, e carece ainda de maturidade e melhoria. ${ }^{1-3,13,14}$

Com efeito, a resposta dos sistemas de saúde a este nível é considerada insuficiente, mesmo que se considere apenas a vertente clínica, dado o défice estrutural da preparação médica nesta área e a escassez dos recursos humanos que lhe estão alocados.

Apesar do paradigma do modelo de saúde clássico vertical, centrado na relação estreita médico - doente, ter evoluído para o conceito horizontal, em que se envolve a criança e a família na tomada de decisões relativamente à sua saúde, a área do neurodesenvolvimento exige ainda um contacto com as equipas de intervenção na comunidade. Neste contexto é de realçar ainda a importância das reuniões pluridisciplinares e da informação escrita detalhada. Estas questões, não sendo acauteladas, motivam elevada insatisfação dos profissionais. ${ }^{14}$

A nível mundial, a especialidade de Pediatria teve necessidade de se diferenciar na área do neurodesenvolvimento e de promover modelos de trabalho multiprofissional em rede, que envolvem outros técnicos de saúde, como os psicólogos, e outras áreas de avaliação e de intervenção terapêutica, bem como as famílias. ${ }^{2,3,11}$

Este trabalho tem como objetivo principal fazer um retrato da realidade assistencial da Pediatria do Neurodesenvolvimento (área diferenciada da Pediatria que tem a responsabilidade neste campo) a nível nacional, de modo a obter dados fundamentados que possam contribuir para a melhoria contínua desta rede de cuidados em Portugal. Adicionalmente, comparou-se a evolução destes indicadores com os obtidos em $2007.4,15$

\section{MATERIAL E MÉTODOS}

Em 2018 e 2019, a Direção da SPND-SPP lançou um questionário online (https://docs.google.com/forms/d/ e/1FAIpQLSdzM9sKZziVdT_EAI3KgY_uPvHvpEkQYi4cNUJLMYxMs5r2Rg/viewform) que foi enviado a todos os hospitais com consultas individualizadas de Pediatria do Desenvolvimento/Neurodesenvolvimento [(doravante utilizaremos neste sentido a terminologia 'Pediatria do Neurodesenvolvimento' (PND)].

Para identificação dos centros hospitalares, hospitais e unidades locais de saúde do Serviço Nacional de Saúde (SNS) com consultas de PND usou-se, em 2018, a listagem acessível no sitio institucional (https://www.sns.gov.pt/ institucional/entidades-de-saude/). O contacto foi realizado por correio eletrónico dirigido ao email institucional dos 43 estabelecimentos hospitalares do SNS, nas condições referidas, no Continente, no arquipélago da Madeira e dos Açores, e a dois hospitais privados com tradição assistencial na área da PND, o que perfez um universo total de 45 instituições de Saúde [listadas no Apêndice 1 (ver Apêndice 1: https://www.actamedicaportuguesa.com/revista/index. php/amp/article/view/13316/Apendice_01.pdf)].

Tendo o estudo como referência o período temporal do biénio 2016-2017, foram colocadas as perguntas que a seguir se referem, relativamente ao movimento e ao tipo assistencial em PND; aos recursos humanos e tempos semanais dispensados a esta área; à capacidade formativa; ao tempo de espera para primeira consulta; e ainda à projeção de necessidades de recrutamento de profissionais a curto prazo:

i) número de consultas de PND (primeiras e total) onde se deveriam englobar as consultas específicas desta área, como por exemplo as de hiperatividade e de autismo, entre outras;

ii) número total de consultas de Pediatria (onde deveriam estar englobadas as de áreas subespecializadas ou diferenciadas, como por exemplo de nefrologia ou endocrinologia, caso existissem);

iii) existência de consultas específicas englobadas na PND, e quais;

iv) existência de internos de Pediatria integrados na consulta de PND e em que contexto formativo;

v) número de pediatras que se dedicava à PND a tempo completo (única atividade assistencial para além do serviço de urgência) ou parcial;

vi) número de psicólogos, terapeutas da fala e ocupacionais, fisioterapeutas, técnicos de psicomotricidade, docentes e técnicos do serviço social que faziam parte destas equipas multiprofissionais;

vii) número de horas de pediatras e dos outros técnicos de saúde dispensados à área de PND, por semana;

viii) número de pediatras com formação em ciclo de estudos especiais em PND, ou com a sua equiparação;

ix) número de utentes em lista de espera para primeira consulta de PND, bem como o respetivo tempo de resolução a 31 de dezembro de 2017;

$x)$ necessidade de recrutar recursos humanos a curto prazo, e quais.

As respostas a estas questões foram fornecidas tendo como fonte oficial os respetivos registos estatísticos hospitalares.

Dada a natureza do estudo, baseado na análise de 
Tabela 1 - Movimento assistencial de consultas de Pediatria do Neurodesenvolvimento em 2016 e 2017, por regiões e ilhas

\begin{tabular}{|c|c|c|c|c|c|c|c|}
\hline Regiões & & Total & Média & DP & Mediana & Mín. & Máx. \\
\hline \multirow{2}{*}{ Lisboa e Vale do Tejo } & 2016 & 33012 & 2201 & 1578 & 2153 & 315 & 6919 \\
\hline & 2017 & 33271 & 2218 & 1568 & 2001 & 339 & 6986 \\
\hline \multirow{2}{*}{ Norte } & 2016 & 30415 & 2172 & 1161 & 1973 & 200 & 4665 \\
\hline & 2017 & 30727 & 2195 & 1175 & 2130 & 225 & 4530 \\
\hline \multirow{2}{*}{ Centro } & 2016 & 26158 & 3270 & 2615 & 2686 & 813 & 8970 \\
\hline & 2017 & 26209 & 3276 & 2531 & 2727 & 842 & 8793 \\
\hline \multirow{2}{*}{ Algarve } & 2016 & 3789 & 1895 & 1298 & 1895 & 977 & 2812 \\
\hline & 2017 & 3704 & 1852 & 1266 & 1852 & 957 & 2747 \\
\hline \multirow{2}{*}{ Madeira } & 2016 & 2007 & 2007 & . & 2007 & 2007 & 2007 \\
\hline & 2017 & 2286 & 2286 & . & 2286 & 2286 & 2286 \\
\hline \multirow{2}{*}{ Alentejo } & 2016 & 1915 & 638 & 305 & 715 & 302 & 898 \\
\hline & 2017 & 1829 & 610 & 361 & 674 & 221 & 934 \\
\hline \multirow{2}{*}{ Açores } & 2016 & 1223 & 612 & 670 & 612 & 138 & 1085 \\
\hline & 2017 & 1789 & 895 & 271 & 895 & 703 & 1086 \\
\hline
\end{tabular}

DP: desvio padrão; max: máximo; min: minimo

indicadores numéricos provenientes de fontes oficiais e em inquérito online respondido de livre e espontânea vontade, e ao facto de não terem sido trabalhados quaisquer dados clínicos pessoais, não houve necessidade de submissão á Comissão de Ética para a Saúde.

\section{RESULTADOS}

Obteve-se resposta das 45 instituições hospitalares questionadas $(100 \%)$, que confirmaram prestar cuidados de PND de um modo diferenciado, estruturado e regular em regime de ambulatório. Destas 45 instituições de saúde, 43 estão integradas no SNS, e duas (4\%) pertencem ao sistema de saúde privado.

Segundo os dados obtidos neste levantamento, o nú- mero total de consultas de PND em 2016 e 2017, foi respetivamente de 98519 (SNS-93 529; 94,9\%) e de 99815 (SNS-94 980; 95,2\%). Nas diferentes regiões e ilhas, nos anos 2016/2017, estes números variaram de valores mínimos de 138/221 a máximos de 8970/8793; com médias \pm um desvio padrão de 2189/2218 $\pm 1662 / 1630$ e medianas de 1971/2001 (Tabela 1). O número total de consultas de Pediatria do Neurodesenvolvimento por regiões e ilhas, e o total em Portugal, ilustra-se na Fig. 1.

A distribuição dos rácios de primeiras consultas de PND em relação ao seu número total variou em Portugal em 2016 de 14,6\% (Sul Alentejo) a 28,5\% (Madeira), e em 2017 de 12,8\% (Madeira) a 19,1\% (Sul Lisboa e Vale do Tejo) (Tabela 2).

Número total de consultas de pediatria neurodesenvolvimento em Portugal por região e ilhas Anos 2016 e 2017

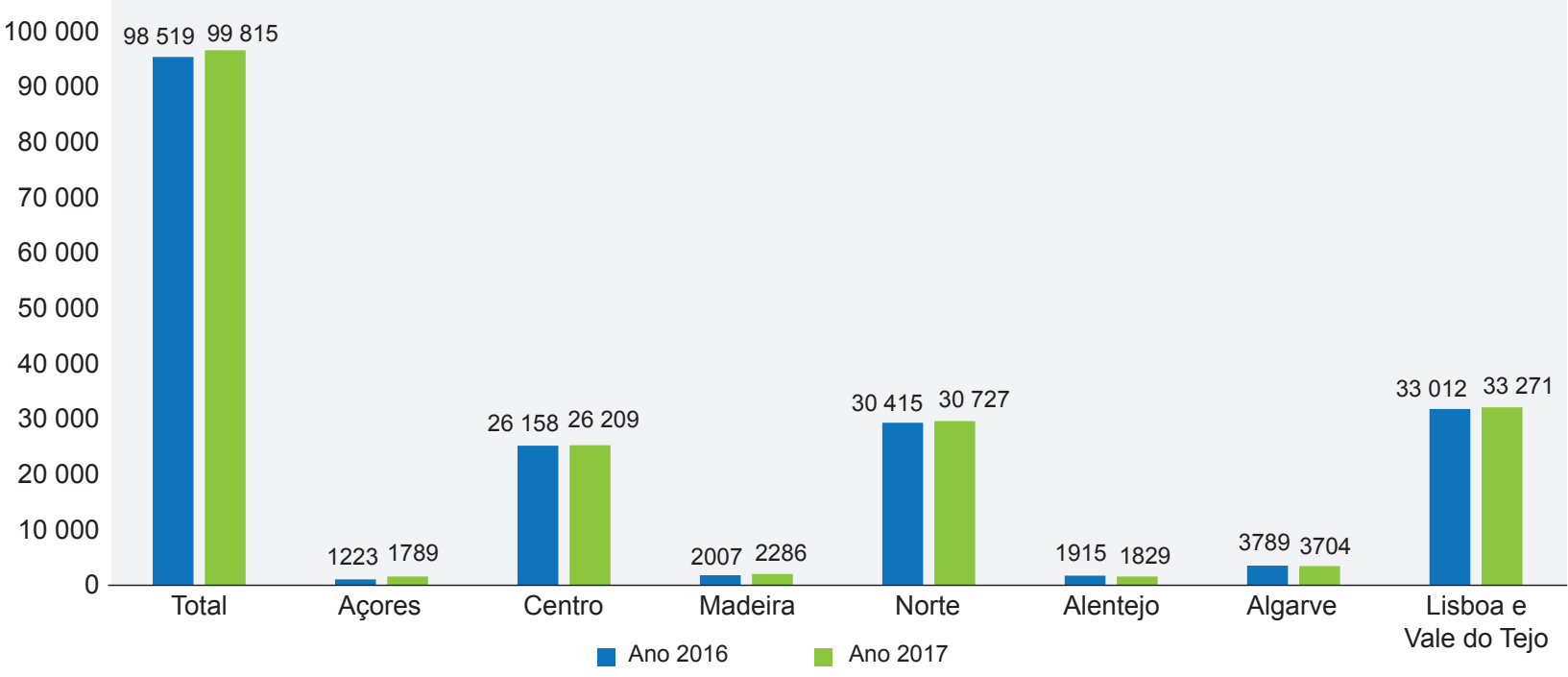

Figura 1 - Distribuição do número total de consultas de Pediatria do Neurodesenvolvimento em Portugal, por regiões e ilhas em 2016 e 2017 
Tabela 2 - Relação entre primeiras consultas de Pediatria do Neurodesenvolvimento e o valor total de consultas de Pediatria do Neurodesenvolvimento por regiões e ilhas em 2016 e 2017

\begin{tabular}{lrrrrrr}
\hline \multirow{2}{*}{ Região } & \multicolumn{3}{c}{ Consultas PND 2016 } & \multicolumn{3}{c}{ Consultas PND 2017 } \\
\cline { 2 - 7 } & Primeiras & Total & Taxa Primeiras/ Total & Primeiras & Total & Taxa Primeiras/ Total \\
\hline Lisboa e Vale do Tejo & 5998 & 33012 & $18,20 \%$ & 6357 & 33271 & $19,10 \%$ \\
Norte & 5445 & 30415 & $17,90 \%$ & 5158 & 30727 & $16,80 \%$ \\
Centro & 4292 & 26158 & $16,40 \%$ & 4208 & 26209 & $16,10 \%$ \\
Algarve & 750 & 3789 & $19,80 \%$ & 686 & 3704 & $18,50 \%$ \\
Madeira & 571 & 2007 & $28,50 \%$ & 292 & 2286 & $12,80 \%$ \\
Alentejo & 280 & 1915 & $14,60 \%$ & 279 & 1829 & $15,30 \%$ \\
Açores & 259 & 1223 & $21,20 \%$ & 329 & 1789 & $18,40 \%$ \\
Total & 17595 & 98519 & $17,86 \%$ & 17309 & 99815 & $17,34 \%$ \\
\hline
\end{tabular}

PND: Pediatria do neurodesenvolvimento

O registo facultado nesses anos pelos hospitais, relativos ao número de consultas de Pediatria no total do país, por regiões e ilhas, bem como a proporção das consultas de PND relativamente ao valor das de Pediatria, foi refletido na Tabela 3. As consultas de PND, relativamente às de Pediatria no seu global, representaram $14,4 \%$, com uma variação, em 2016, entre 8,5\% nos Açores e 24,5\% na região Centro; em 2017, o valor variou de 12,3\% na região de Lisboa e Vale do Tejo a $24,2 \%$ no Centro.

Relativamente ao tipo de consultas que ofereciam à população, 31 estruturas hospitalares dispunham de consultas gerais de PND onde seguiam todas as patologias desta área. Nos restantes 14 (31\%) foram criadas consultas específicas. De entre estas, oito hospitais dispunham de consultas de hiperatividade, oito de risco biológico, sete de autismo, quatro de trissomia 21, quatro de dificuldades de aprendizagem, três de paralisia cerebral, uma de neonatologia e uma de defeitos do tubo neural.

Quanto à disponibilidade desta área médica para formação em contexto de internatos de Pediatria, nove hospitais proporcionavam este estágio opcional. Em 27 (60\% do total dos hospitais), por rotina, os internos de pediatria beneficiavam de formação nas consultas de PND, onde dispunham de tempo de estágio (embora não fazendo parte obrigatória do programa de formação específica em pediatria). Em nove, essa possibilidade não estava disponível.

O número de pediatras dedicados a tempo inteiro à PND era de 56 e a tempo parcial de 100, dedicando a esta área, no total, 2279 horas por semana. Destes pediatras, vinte e nove eram detentores do ciclo de estudos especiais em PND.

No que diz respeito à integração de outros profissionais de saúde nestas equipas, apresenta-se entre parêntesis o seu número e a quantidade de horas que dedicavam por semana à PND: psicólogos (82; 1447), terapeutas da fala (64; 1325), fisioterapeutas $(40 ; 522)$, terapeutas ocupacionais $(28 ; 648)$, técnicos de psicomotricidade $(16 ; 399)$, docentes $(30 ; 684)$ e técnicos do serviço social $(32 ; 401)$ (Tabelas 4 e 5).

A 31 de dezembro de 2017, uma média de 101 crianças aguardavam uma consulta de PND, variando este valor entre 4 e 387 . A taxa de resolução da lista de espera, em média de três meses, variava de 1 a 10 (Tabela 6).

A necessidade de contratação a curto prazo de pediatras para a área de PND foi manifestada por 14 hospitais, que no seu conjunto recrutariam 15 profissionais (oito para o Sul, quatro para o Norte e três para o Centro). Para além destes foram solicitados, por ordem decrescente, psicólogos (25), terapeutas da fala (14), terapeutas ocupacionais (10), técnicos de psicomotricidade (9), fisioterapeutas (6) e docentes (1).

\section{DISCUSSÃO}

No enquadramento mundial, em que assistimos a um aumento da prevalência das perturbações do neurodesenvolvimento e consequente procura de cuidados de saúde

Tabela 3 - Proporção entre as consultas de Pediatria do Neurodesenvolvimento e as de Pediatria em Portugal em 2016 e 2017

\begin{tabular}{lrrrrrr}
\hline Região & $\begin{array}{r}\mathbf{N}^{\circ} \text { Consultas } \\
\text { Ped 2016 }\end{array}$ & $\begin{array}{r}\mathbf{N}^{\circ} \text { Consultas } \\
\text { PND 2016 }\end{array}$ & $\begin{array}{r}\text { PND/ Ped 2016 } \\
(\%)\end{array}$ & $\begin{array}{r}\mathbf{N}^{\circ} \text { Consultas } \\
\text { Ped 2017 }\end{array}$ & $\begin{array}{r}\mathbf{N}^{\circ} \text { Consultas } \\
\text { PND 2017 }\end{array}$ & $\begin{array}{r}\text { PND/Ped 2017 } \\
\text { (\%) }\end{array}$ \\
\hline Lisboa e Vale do Tejo & 265775 & 33012 & $12,40 \%$ & 270364 & 33271 & $12,30 \%$ \\
Norte & 258529 & 30415 & $11,80 \%$ & 259131 & 30727 & $11,90 \%$ \\
Centro & 106949 & 26158 & $24,50 \%$ & 108480 & 26209 & $24,20 \%$ \\
Algarve & 15871 & 3789 & $23,90 \%$ & 15503 & 3704 & $23,90 \%$ \\
Madeira & 13837 & 2007 & $14,50 \%$ & 12337 & 2286 & $18,50 \%$ \\
Alentejo & 16560 & 1915 & $11,60 \%$ & 15523 & 1829 & $11,80 \%$ \\
Açores & 14351 & 1223 & $8,50 \%$ & 13751 & 1789 & $13,00 \%$ \\
Total & 691872 & 98519 & $14,24 \%$ & 695089 & 99815 & $14,36 \%$ \\
\hline
\end{tabular}

Ped: Pediatria; PND: Pediatria do neurodesenvolvimento 
Tabela 4 - Profissionais que integravam as equipas de PND em 2017, distribuídos por regiões e ilhas, relativamente ao número de elementos e de horas por semana dedicados à área

\begin{tabular}{|c|c|c|c|c|c|c|c|c|c|}
\hline Região & Ped TC & Ped TP & Ped H/S & Psi & Psic H/S & TF & TF H/S & Fis & Fis $\mathrm{H} / \mathrm{S}$ \\
\hline Lisboa e Vale do Tejo & 20 & 33 & 898 & 32 & 710 & 22 & 488 & 9 & 115 \\
\hline Centro & 16 & 18 & 479 & 17 & 300 & 15 & 394 & 4 & 70 \\
\hline Norte & 15 & 33 & 631 & 22 & 204 & 13 & 126 & 18 & 119 \\
\hline Algarve & 4 & 3 & 138 & 2 & 35 & 3 & 55 & 2 & 70 \\
\hline Alentejo & 1 & 7 & 76 & 3 & 42 & 3 & 79 & 1 & 35 \\
\hline Açores & 0 & 3 & 17 & 4 & 86 & 6 & 113 & 5 & 78 \\
\hline Madeira & 0 & 3 & 40 & 2 & 70 & 2 & 70 & 1 & 35 \\
\hline Total & 56 & 100 & 2279 & 82 & 1447 & 64 & 1325 & 40 & 522 \\
\hline
\end{tabular}

Fis: fisioterapeutas. H/S: horas por semana; Ped: pediatras; Psi: psicólogos; TC: tempo completo; TF: terapeutas falas; TP: tempo parcial

Tabela 5 - Profissionais que integravam as equipas de PND em 2017, distribuídos por regiões e ilhas, relativamente ao número de elementos e de horas por semana dedicados à área

\begin{tabular}{lrrrrrrrr}
\hline Região & TO & TO H/S & TPsi & TPsi H/S & Doc & Doc H/S & TSS & TSS H/S \\
\hline Lisboa e Vale do Tejo & 6 & 183 & 9 & 204 & 8 & 180 & 6 & 43 \\
Norte & 10 & 92 & 2 & 35 & 4 & 14 & 14 & 132 \\
Centro & 5 & 175 & 2 & 55 & 15 & 441 & 6 & 101 \\
Algarve & 3 & 105 & 0 & 0 & 1 & 4 & 1 & 12 \\
Alentejo & 0 & 0 & 1 & 35 & 0 & 1 & 10 & 2 \\
Açores & 3 & 58 & 2 & 70 & 1 & 35 & 4 \\
Madeira & 1 & 35 & 0 & 0 & 30 & 684 & 32 \\
Total & 28 & 648 & 16 & 399 & 401 \\
\hline
\end{tabular}

Doc: docentes; H/S: horas por semana; TO: terapeutas ocupacionais; TC: tempo completo; TP: tempo parcial; Tpsi: técnicos de psicomotricidade; TSS: técnicos de serviço social

adequados, Portugal vem incorporando esta realidade. Em dez anos (2007 a 2017), o número total de consultas hospitalares de Pediatria do Neurodesenvolvimento aumentou de $38238^{4}$ para 99815 , o que representa quase o triplo: 2,6 vezes (Tabela 7 ).

Este fenómeno poderia estar contextualizado no aumento, em termos globais, das consultas médicas hospitalares a que se vem assistindo, particularmente na especialidade de Pediatria. No entanto, neste período temporal, fazendo uma análise deste movimento, e tendo por base referências oficiais, ${ }^{16,17}$ o número de consultas de pediatria sofreu um incremento de $7 \%$, o que está bastante abaixo do verificado nas consultas de PND, como demonstramos no nosso trabalho, reforçando o seu real aumento absoluto e relativo.

No estudo anterior, em 2007, a taxa global calculada de consultas de PND relativamente ao total das de Pediatria correspondia a $11 \%$, sendo na região Centro a mais elevada, (próxima de 17\%). ${ }^{4}$ Atualmente, essa relação global subiu três pontos percentuais, ultrapassando agora os $14 \%$. Na região Centro continua a manter-se a taxa mais elevada, atingido presentemente as consultas de PND quase um quarto das de Pediatria (Tabela 3). De salientar que esta elevada proporção, comparativamente ao resto do País, poderá ter como explicação o facto de a região Centro ser a área do país onde o número de consultas hospitalares de PND no sistema privado menos se fará notar, o que pode, relativamente às outras regiões, fazer aumentar esta resposta hospitalar no contexto do SNS.
Em 2017, o número de especialistas em Pediatria dedicados a esta área era de 56 a tempo inteiro e 100 a tempo parcial. Os dados disponíveis permitem-nos concluir que o número de pediatras afetos à área da Pediatria do Neurodesenvolvimento quase duplicou (de 82 em 2007 para 156 em 2017). Em 2007, a relação de pediatras dedicados à PND (82) relativamente ao total de pediatras (1479) era de $5,5 \%{ }^{18}$. Em 2017, essa proporção subiu para $7,5 \%$ (156/2085) - Tabela 7.

Nesse mesmo ano, a amostra nacional estudada, revelou que o número de horas de trabalho da especialidade hospitalar de pediatria alocada à área de PND, era de 2279 horas por semana. Considerando que o corpo clínico hospitalar trabalha cerca de 45 semanas por ano (excetua-se o

Tabela 6 - Número de crianças em lista de espera para primeira consulta de PND em Portugal a 31 dezembro de 2017

\begin{tabular}{|c|c|c|c|}
\hline & & $\begin{array}{r}\mathbf{N}^{\circ} \text { crianças lista } \\
\text { espera } \\
(31 / 12 / 2017)\end{array}$ & $\begin{array}{r}\text { Taxa resolução lista } \\
\text { espera } \\
(31 / 12 / 2017) \text {, meses }\end{array}$ \\
\hline \multicolumn{2}{|l|}{ Média } & 101 & 3 \\
\hline \multicolumn{2}{|l|}{ Mediana } & 73 & 3 \\
\hline \multicolumn{2}{|c|}{ Desvio padrão } & 95 & 2 \\
\hline \multicolumn{2}{|l|}{ Minimo } & 4 & 1 \\
\hline \multicolumn{2}{|l|}{ Máximo } & 387 & 10 \\
\hline \multirow{3}{*}{ Percentis } & 25 & 40 & 1,6 \\
\hline & 50 & 73 & 3,0 \\
\hline & 75 & 112 & 3,3 \\
\hline
\end{tabular}


Tabela 7 - Dados comparativos entre os anos 2007 e 2017

\begin{tabular}{lrrr}
\hline Anos de estudo & $\begin{array}{r}\mathbf{N}^{\circ} \text { total } \\
\text { Consultas PND }\end{array}$ & $\begin{array}{r}\mathbf{N}^{\circ} \text { Pediatras } \\
\text { dedicados PND/ total pediatras } \\
(\%)\end{array}$ & $\begin{array}{r}\text { Mediana de crianças em lista espera a } \\
\text { 31 de dezembro para consulta PND } \\
\text { (mediana de espera em meses) }\end{array}$ \\
\hline \multirow{2}{*}{2007} & 38.238 & $82 / 1479$ & 185 \\
& & $(5,5 \%)$ & 101 \\
2017 & 99.815 & $156 / 2085$ & $(3)$ \\
\hline
\end{tabular}

PND: Pediatria do neurodesenvolvimento

período de férias e licença para formação sem perda de remuneração, entre outras), conclui-se que o número de horas calculado por ano reportaria a 102555 horas. Partindo do princípcio de que o número total de consultas de PND nesse ano, em Portugal, foi de 99 815, poderemos concluir que o tempo médico dedicado a cada consulta foi em média de uma hora. No regulamento recentemente publicado pela Ordem dos Médicos, relativo aos tempos padrão das consultas, o Colégio de Pediatria recomenda que as consultas de PND tenha uma duração de 60 minutos para primeiras e 45 para subsequentes. ${ }^{19}$ Tendo por base estes tempos, e considerando o número de consultas de PND neste levantamento (99 815), e o peso relativo das primeiras versus subsequentes (17 309/82 506), este movimento consumiria 79188 horas por ano. Nesta projeção, restaria menos de um quarto deste tempo (23 367/102 555 horas; $22.8 \%$ ) para alocar a reuniões, contactos com o exterior e elaboração de relatórios, o que seria manifestamente insuficiente.

Vários estudos evidenciam insatisfação e desmotivação profissional dos pediatras que se dedicam a esta área. ${ }^{14}$ Uma das razões é a atribuição insuficiente de horas para tarefas pós consulta, como também se verifica em Portugal. A outra é a reclamação de falta de formação específica na área. ${ }^{20}$ Num estudo recente da Academia Americana de Pediatria, 65\% dos questionados referia ter falta de preparação para intervir nas patologias do neurodesenvolvimento. ${ }^{20}$

Em Portugal, estas questões vêm sendo acauteladas, embora ainda a carecer de melhoria.

O reforço do quadro médico atribuído a esta área tem sido evidente, a par de um esforço de formação específica na área do neurodesenvolvimento, quer a nível do internato de Pediatria, quer numa fase posterior de diferenciação.
De facto, o nosso estudo revelou que um quinto dos hospitais públicos (9/43) têm idoneidade formativa para proporcionar estágio opcional nesta área, sendo que em 27 $(60 \%)$, os internos de pediatria frequentam habitualmente as consultas de PND (embora no programa de formação atual de pediatria a formação nesta área seja opcional, passando a ser obrigatória na atualização em curso).

A formação diferenciada de dois anos nesta área, através da frequência do Ciclo de Estudos Especiais (CEE) para especialistas em Pediatria, existe em Portugal desde $2009,{ }^{15}$ o que permitiu que 29 deste 156 pediatras sejam detentores do CEE em PND (18,6\%).

A pluridisciplinaridade hospitalar e a ligação destes profissionais aos da comunidade onde as crianças se encontram integradas são especificidades da PND e oneram esta área em termos de recursos humanos e de tempos diretos (consulta) e indiretos (pós consulta), como já foi salientado.

No levantamento de 2007, a escassez de recursos de saúde atribuídos à PND na área dos profissionais não médicos também era muito evidente, tendo-se verificado um elevado número de solicitações para o seu reforço. $\mathrm{Na}$ Tabela 8 ilustra-se o número de profissionais existentes em 2007, a solicitação de contratações então elencada e os registos de profissionais atualmente existentes. Pode verificar-se que o aumento foi positivo, em todas as áreas, sobretudo nas de pediatria (acréscimo de 90\%), psicologia $(26 \%)$, terapia da fala (52\%) e fisioterapia (21\%). Assistiu-se a uma redução de $3 \%$ no número de terapeutas ocupacionais que eventualmente deram lugar aos técnicos de psicomotricidade, não atribuídos em 2007. O número de docentes aumentou $11 \%$, mas ficou aquém do programado, assim como os profissionais de serviço social que sofreram uma redução de $5 \%$.

Das solicitações a curto prazo que neste levantamento

Tabela 8 - Profissionais das equipas de Pediatria do Neurodesenvolvimento em 2007 e 2017, existentes e solicitados

\begin{tabular}{|c|c|c|c|c|c|}
\hline Profissionais & $\begin{array}{r}\text { Existentes } \\
2007\end{array}$ & $\begin{array}{r}\text { Solicitados } \\
2007\end{array}$ & $\begin{array}{r}\text { Existentes } \\
2017\end{array}$ & $\begin{array}{r}\text { Acréscimo } \\
2007 / 2017(\%)\end{array}$ & $\begin{array}{r}\text { Solicitados curto } \\
\text { prazo }\end{array}$ \\
\hline Pediatria & 82 & 34 & 156 & $+90 \%$ & 15 \\
\hline Psicologia & 65 & 21 & 82 & $+26 \%$ & 25 \\
\hline Terapia fala & 42 & 20 & 64 & $+52 \%$ & 14 \\
\hline Fisioterapia & 33 & 8 & 40 & $+21 \%$ & 6 \\
\hline Terapia ocupacional & 29 & 14 & 28 & $-3 \%$ & 10 \\
\hline Psicomotricidade & - & - & 16 & & 9 \\
\hline Docência & 27 & 20 & 30 & $+11 \%$ & 1 \\
\hline Serviço social & 38 & 6 & 36 & $-5 \%$ & - \\
\hline
\end{tabular}

Nota: Por limitação de dados de confiança relativamente ao regime de trabalho não se discrimina se é de tempo completo ou parcial 
foram inventariadas (Tabela 8), mantém-se, sobretudo, a necessidade de reforço do quadro de Pediatria, de Psicologia e de Terapia da Fala, seguido pela Terapia Ocupacional, Psicomotricidade e Fisioterapia. Estes profissionais constituem o núcleo central das equipas pluridisciplinares hospitalares em Portugal, à semelhança do preconizado noutros países. ${ }^{2,5-7}$

Apesar do aumento expressivo de profissionais dedicados à área da Pediatria do Neurodesenvolvimento a nível do SNS (somente dois hospitais pertenciam ao sistema privado), e do número de consultas quase ter triplicado, a resposta às necessidades demonstradas pela população pediátrica ainda não está devidamente acautelada. Em dezembro de 2017, a mediana do número de crianças em lista de espera era de 101 crianças (variando de 4 a 387), as quais aguardavam por primeira consulta entre um a dez meses (mediana três) - Tabela 6. Não sendo estes dados os ideais, são francamente melhores que os registados há dez anos, em que a mediana de crianças em lista de espera há seis meses (mediana) era de 185 (Tabela 7). ${ }^{4}$

O panorama da criança doente alterou-se nas últimas décadas, com as patologias do neurodesenvolvimento a figurarem atualmente no topo da lista das doenças crónicas, como sendo as mais frequentes. Se no passado o protótipo da criança doente era a que sofria de um quadro infecioso e até de desnutrição, hoje será o da que sofre de perturbação do neurodesenvolvimento. ${ }^{5}$ Neste âmbito, destacam-se os problemas do défice de atenção e hiperatividade, da perturbação do desenvolvimento intelectual, da perturbação do espetro do autismo e das condições clínicas anteriormente fatais associadas a elevado risco de sequelas de funcionamento cerebral nos sobreviventes, como a grande prematuridade, a doença oncológica, neurológica degenerativa e as doenças crónicas em geral. ${ }^{5,21}$ Deste modo, as entidades hospitalares de saúde vêm-se organizando no sentido de responder às necessidades da população.

Neste estudo podemos constatar que nos hospitais nacionais referidos está refletida esta diversidade de respostas na área da PND. Em cerca de um terço dos nossos hospitais (14/45) foram criadas consultas específicas no âmbito das patologias do neurodesenvolvimento, que claramente refletem as perturbações mais comuns, ou mais complexas, ou grupos clínicos emergentes. Oito hospitais dispõem de consulta diferenciada de hiperatividade, oito de risco biológico, e sete de autismo, o que permite responder a patologias mais específicas de um modo normalizado. ${ }^{1}$

\section{CONCLUSÃO}

Este é um trabalho de âmbito nacional, com uma taxa de respostas de $100 \%$, baseado em registos oficiais hospitalares e dados fornecidos por colegas diretamente envolvidos na área, que permite demonstrar que a resposta hospitalar do SNS em Pediatria do Neurodesenvolvimento aumentou de um modo significativo. O movimento de consultas tripli- cou. Em termos globais, o número de profissionais, numa perspetiva pluridisciplinar, aumentou de um modo evidente, sendo inferior o número de crianças em lista de espera e o tempo que aguardam pela consulta. Na formação dos profissionais médicos há factos que objetivam progressos, dado que cerca de um quinto dos pediatras dedicados a esta área são detentores do CEE em PND, e há dez anos essa formação não existia. Apesar de se reconhecer objetivamente esta evolução positiva, ainda assim, subsistem eixos para melhoria. Aos profissionais médicos apenas restava menos de um quarto do seu horário para trabalho após a consulta, o que é claramente insuficiente nesta área da saúde, e promotor de insatisfação.

Nessa perspetiva, os hospitais fizeram a devida reflexão, continuando a solicitar o reforço de profissionais para estas equipas, com um foco mais expressivo na área da Psicologia.

Contudo, é importante acautelar que esta é uma complexa área de doença crónica, que se manifesta na idade pediátrica, mas que, na maioria dos casos, permanecerá e continuará a necessitar de cuidados de saúde e psicossociais na idade adulta. ${ }^{2}$

\section{AGRADECIMENTOS}

A Sociedade de Pediatria do Neurodesenvolvimento da Sociedade Portuguesa de Pediatria está muito grata às Direções e Equipas do Neurodesenvolvimento dos Hospitais elencados no Apêndice 1 (ver Apêndice 1: https://www. actamedicaportuguesa.com/revista/index.php/amp/article/ view/13316/Apendice_01.pdf), que de um modo altruísta, com alta qualidade e eficiência, colaboraram neste estudo de relevância elevada para a organização da Pediatria nacional.

\section{PROTEÇÃO DE PESSOAS E ANIMAIS}

Os autores declaram que os procedimentos seguidos estavam de acordo com os regulamentos estabelecidos pelos responsáveis da Comissão de Investigação Clínica e Ética e de acordo com a Declaração de Helsínquia da Associação Médica Mundial actualizada em 2013.

\section{CONFIDENCIALIDADE DOS DADOS}

Os autores declaram ter seguido os protocolos do seu centro de trabalho acerca da publicação de dados.

\section{CONFLITOS DE INTERESSE}

Os autores declaram não ter conflitos de interesses relacionados com o presente trabalho.

\section{FONTES DE FINANCIAMENTO}

Este trabalho não recebeu qualquer tipo de suporte financeiro de nenhuma entidade no domínio público ou privado. 


\section{REFERÊNCIAS}

1. Soares NS, Baum RA, Frick KD. Improving developmental-behavioral pediatric dare workflow. J Dev Behav Pediatr. 2015;36:45-52.

2. McDowell M, Lesslie J. Neurodevelopmental-behavioural paediatrics. Curr Opin Pediatr. 2019;31:797-806.

3. McDowell M. Child with multiple problems: clinical complexity and uncertainty. J Paediatr Child Health. 2018;54:1084-9.

4. Oliveira G, Duque F, Duarte C, Melo F, Teles L, Brito M, et al. Pediatria do Neurodesenvolvimento. Levantamento nacional de recursos e necessidades. Acta Pediatr Port. 2012;43:1-7.

5. Halfon N, Houtrow A, Larson K, Newacheck PW. Changing landscape childhood disability. Future Child. 2012;22:13-42.

6. Houtrow AJ, Larson K, Olson LM, Newacheck PW, Halfon N. Changing trends of childhood disability, 2001-2011. Pediatrics. 2014;134:530-8.

7. Boyle CA, Boulet S, Schieve LA, Cohen RA, Blumberg SJ, YearginAllsopp M, et al. Trends in the prevalence of developmental disabilities in US children, 1997-2008. Pediatrics. 2011;127:1034-42.

8. Carter SM, Rogers W, Heath I, Degeling C, Doust J, Barratt. The challenge of overdiagnosis begins with its definition. BMJ. 2015;350:h869.

9. Brodersen J. How to conduct research on overdiagnosis. A keynote paper from the EGPRN May 2016, Tel Aviv. Eur J Gen Pract. 2017;23:7882.

10. American Psychiatric Association. The Diagnostic and Statistical. Manual of Mental Disorders. $5^{\text {th }}$ ed. Arlington: APA Publishing; 2013.

11. D'Souza H, Karmiloff-Smith A. Neurodevelopmental disorders. Interdiscip Rev Cogn Sci. 2017;8.
12. Doernberg E, Hollander E. Neurodevelopmental disorders (ASD and ADHD): DSM-5, ICD-10, and ICD-11. CNS Spectr. 2016;21:295-9.

13. McMillan JA, Land M Jr, Leslie LK. Pediatric residency education and the behavioral and mental health crisis: a call to action. Pediatrics. 2017;139:e20162141.

14. Bridgemohan C, Bauer NS, Nielsen BA, DeBattista A, Ruch-Ross HS, Paul LB, et al. A workforce survey on developmental-behavioral pediatrics. Pediatrics. 2018;141:e20172164.

15. Oliveira G. A Pediatria do Neurodesenvolvimento, o que é? Acta Pediatr Port. 2011,42:61-3.

16. Instituto Nacional de Estatística. Anuário Estatístico de Portugal 2009. Saúde 145-169. Lisboa: INE; 2010.

17. Ministério da Saúde. Relatório anual do acesso 2018. Lisboa: MS; 2018.

18. Pordata. Médicos: não especialistas e especialistas por especialidade. [consultado 2019 set 09]. Disponível em: https://www.pordata.pt/ Portugal/M\%c3\%a9dicos $+n \% c 3 \% a 30+$ especialistas+e+especialistas+ por+especialidade.

19. Regulamento $n^{\circ} 724 / 2019$. Diário da República, II Série, n. ${ }^{\circ} 178$ (2019/09/17). p.103-18.

20. Horwitz SM, Storfer-Isser A, Kerker BD, Szilagyi M, Garner A, O'Connor $K G$, et al. Barriers to the identification and management of psychosocial problems: changes from 2004 to 2013. Acad Pediatr. 2015;15:613-20.

21. Moreira MC, Gomes R, Calheiros de Sá MR. Chronic diseases in children and adolescents: a review of the literature. Cien Saude Colet. 2014;19:2083-94. 